\title{
A Global Game with Strategic Substitutes and Complements: Note
}

By

\author{
Eric J. Hoffmann* and Tarun Sabarwal ${ }^{\dagger}$
}

\begin{abstract}
In a 2007 paper, "A global game with strategic substitutes and complements", by Karp, L., I.H. Lee, and R. Mason, Games and Economic Behavior, 60(1), 155-175, an argument is made to show existence of Bayesian-Nash equilibrim in global games that may include both strategic substitutes and complements. This note documents a gap in the proof of that statement and presents an alternative proof for a finite player version of their model.
\end{abstract}

JEL Numbers: C70, C72

Keywords: Global games, strategic complements, strategic substitutes, monotone games, equilibrium selection

First Draft: April 2014

This Version: February 13, 2015

\footnotetext{
*Department of Economics, University of Kansas. Email: eric3353@ku.edu. Corresponding author.

${ }^{\dagger}$ Department of Economics, University of Kansas. Email: sabarwal@ku.edu.
} 


\section{Introduction}

The global games method, as proposed by Carlsson and Van Damme (1993), shows that in some classes of games with strategic complements, the issue of multiple equilibria can be resolved with a slight relaxation of common knowledge about an underlying fundamental. ${ }^{1}$ Carlsson and Van Damme (1993) work with a 2-player, 2-action coordination game. Morris and Shin (2003) extend this to the case of infinitely many players. Both show that when players observe private, noisy signals about a fundamental, then iterated deletion of strictly dominant strategies produces an "essentially unique" Bayesian-Nash equilibrium strategy, which is monotone in the signal.

Karp, Lee, and Mason (2007) (KLM from here on) investigate a model similar to that of Morris and Shin (2003), but allow for congestion effects by introducing varying degrees of strategic substitutes in the model. ${ }^{2}$ In general, games with strategic substitutes are harder to work with. In particular, a finite-player game with strategic substitutes does not necessarily have a pure-strategy Nash equilibrium (confer Roy and Sabarwal (2012)). Moreover, in a finite-player game with both strategic complements and substitutes, a pure-strategy Nash equilibrium does not necessarily exist (confer Monaco and Sabarwal $(2015))$.

KLM consider a specialized version of games that may have both strategic complements and substitutes and focus on symmetric Bayesian-Nash equilibrium. One of their results (Proposition 1, page 161 in their paper) is that in the Bayesian game associated with the underlying complete information game, a Bayesian-Nash equilibrium always exists. In this note, we document a gap in their proof of this statement and we present an alternative proof of existence of equilibrium for an (arbitrarily large but) finite player

\footnotetext{
${ }^{1}$ Recall that games with strategic complements formalize the idea that observing a higher action from an opponent makes it more beneficial for a given player to also choose a higher action. In these cases, multiple equilibria arise easily.

${ }^{2}$ Recall that games with strategic substitutes formalize the idea that observing a higher action from an opponent makes it more beneficial for a given player to choose a lower action.
} 
version of their model. This may be motivated as an approximation of the model with a continuum of players, as usual.

\section{KLM's Model}

There is a continuum of agents of mass 1 , indexed $i \in[0,1]$, each considering an action in $\{0,1\}$. There is a payoff-relevant state given by $\theta \in \mathbb{R}$. Each player $i$ observes a private signal of the state $x_{i}=\theta+\eta_{i}$, where $\eta_{i}$ is a random variable drawn uniformly from $[-\epsilon, \epsilon], \epsilon>0$, and then chooses an action. KLM define the strategy space as the set of all distributional strategies $y_{i}$ so that for any signal $x \in \mathbb{R}, y_{i}(x)$ gives the probability of choosing action $1 .^{3}$ The strategy space for a player is denoted $Y$ and is endowed with the sup-norm metric: for every $y, y^{\prime} \in Y$,

$$
d\left(y, y^{\prime}\right)=\sup _{x \in \mathbb{R}}\left|y(x)-y^{\prime}(x)\right|
$$

KLM assume that for a given player $i$, the actions of other players affect player $i$ only through the proportion of other players that play 1 . This proportion is denoted $\alpha \in[0,1]$. In the complete information setting, utility depends on the underlying state $\theta$ and on $\alpha$ as follows

$$
U_{i}(\theta, \alpha)=\theta+f(\alpha)
$$

where $f:[0,1] \rightarrow \mathbb{R}$ is an interaction function which captures the congestion effect. The function $f$ is assumed to be an analytic function. When $f$ is everywhere increasing, the game is one of strategic complements. When $f$ is decreasing, there are strategic substitutes. When $f$ is neither always increasing nor always decreasing, we have both strategic complements and substitutes, depending on the slope of $f$.

\footnotetext{
${ }^{3}$ KLM term these distributional strategies, but more precisely, they are behavioral strategies. As noted in Milgrom and Weber (1985), there is a simple rule to transform one into another. In this section, to aid reference to KLM, we shall defer to their terminology, although regardless of terminology, the set of such objects is shown below to be not sup-norm compact.
} 
With incomplete information, KLM restrict attention to symmetric equilibrium. In particular, if each of player $i$ 's opponents plays the same strategy $y$, then the proportion of individuals choosing action 1 given realization of state $\theta$ is

$$
\alpha(\theta, y)=\frac{1}{2 \epsilon} \int_{\theta-\epsilon}^{\theta+\epsilon} y(z) d z
$$

When player $i$ observes signal $x$, expected utility of player $i$ from playing $y_{i}$ given others are playing $y$ is

$$
u_{i}\left(x, y_{i}, y\right)=\left(\frac{1}{2 \epsilon} \int_{x-\epsilon}^{x+\epsilon}(\theta+f(\alpha(\theta, y))) d \theta\right) y_{i}(x) .
$$

In this setting, KLM state the following proposition.

Proposition 1 (KLM, page 161). In the game of incomplete information, there is an equilibrium in distributional strategies.

KLM provide a proof of Proposition 1 on page 168 in their paper, invoking the fixed point theorem in Glicksberg (1952). Glicksberg's theorem requires compact strategy spaces. KLM use the following paragraph to justify that the strategy space is compact in the sup-norm topology:

From Milgrom and Weber (1985, Theorem 1), each player's set of distributional strategies is a compact, convex metric space in the weak topology. In order to establish continuity of expected payoffs, we shall use the sup-norm metric on the set of distributional strategies. The topology induced by this metric is necessarily stronger than the weak topology, since the weak topology ensures continuity only of linear functionals. Hence each player's set of distributional strategies remains compact under this metric.

The last sentence, starting with "Hence each player's...", is not justified by the previous statements. Certainly, a topology stronger than the weak topology may ensure continuity of functions other than just linear functionals. But that means more open sets, and hence 
more open covers to test for compactness. Therefore, compactness is harder to satisfy under stronger topologies. We present a class of counter-examples to the claim that the set of strategies used in KLM is compact in the sup-norm topology.

Claim 1. The set $Y$ of player i's strategies is not compact in the sup-norm metric.

Proof. Suppose to the contrary that $Y$ is compact in the sup-norm metric. Fix $\epsilon \in\left(0, \frac{1}{2}\right)$ arbitrarily, and consider the open cover of $Y$ given $\{B(y, \epsilon): y \in Y\}$, where each $B(y, \epsilon)$ is the open ball of radius $\epsilon$ according to the sup-norm metric. Let $\left\{B\left(y^{n}, \epsilon\right): n=1, \ldots N\right\}$ be a finite subcover. Fix an arbitrary collection of points $\left\{x_{n}\right\}_{n=1}^{N}$ in $\mathbb{R}$ and a strategy $z_{i} \in Y$ that satisfies

$$
z_{i}\left(x_{n}\right) \in \begin{cases}{\left[0, y_{i}^{n}\left(x_{n}\right)-\epsilon\right)} & \text { if } y_{i}^{n}\left(x_{n}\right)>\epsilon, \text { and } \\ \left(y_{i}^{n}\left(x_{n}\right)+\epsilon, 1\right] & \text { if } y_{i}^{n}\left(x_{n}\right) \leq \epsilon\end{cases}
$$

Then for each $n$, either $z_{i}\left(x_{n}\right)>y_{i}^{n}\left(x_{n}\right)+\epsilon$ or $z_{i}\left(x_{n}\right)<y_{i}^{n}\left(x_{n}\right)-\epsilon$, so that $\left|z_{i}\left(x_{n}\right)-y_{i}^{n}\left(x_{n}\right)\right| \geq$ $\epsilon$. Therefore, for each $n$,

$$
d\left(z_{i}, y_{i}^{n}\right)=\sup _{x \in \mathbb{R}}\left|z_{i}(x)-y_{i}^{n}(x)\right| \geq\left|z_{i}\left(x_{n}\right)-y_{i}^{n}\left(x_{n}\right)\right| \geq \epsilon,
$$

so that for each $n, z_{i} \notin B\left(y_{i}^{n}, \epsilon\right)$. This implies that $z_{i} \notin \bigcup_{n=1}^{N} B\left(y_{i}^{n}, \epsilon\right)$, a contradiction to the fact that $\left\{B\left(y^{n}, \epsilon\right): n=1, \ldots N\right\}$ is a finite subcover. Consequently, $Y$ is not compact in the sup-norm metric.

In their discussion (KLM, page 160), KLM indicate that the existence proof in Milgrom and Weber (1985) does not apply because of a violation of the "absolutely continuous information" condition, but that it can be overcome using the simplifications in their model. In particular, they show that expected utility is continuous in the sup-norm metric (page 168-169) and combined with a strategy space that is compact in the supnorm metric and is convex, Glicksberg's theorem may be applied using the sup-norm metric. The claim above shows that the strategy space is not compact in the sup-norm metric, and therefore, the approach in KLM is not entirely correct. 
In the next section, we prove existence of equilibrium in a finite player version of the global game in KLM, using a result due to Balder (1988).

\section{Equilibrium Existence}

Let's recall the setting in Balder (1988). Consider a game with finitely many players, indexed $i=1, \ldots, N$. Each player $i$ has a type space $T_{i}$ with a sigma-algebra $\mathcal{T}_{i}$, and a compact, metric action space $A_{i}$ with the Borel sigma-algebra $\mathcal{A}_{i}$. The product spaces are denoted $T=\times_{i=1}^{N} T_{i}, \mathcal{T}=\times_{i=1}^{N} \mathcal{T}_{i}, A=\times_{i=1}^{N} A_{i}$, and $\mathcal{A}=\times_{i=1}^{N} \mathcal{A}_{i}$. Each player $i$ has a payoff function $U_{i}: T \times A \rightarrow \mathbb{R}$. Let $\eta$ be a probability measure on $(T, \mathcal{T})$ that governs the random behavior of joint information and for each $i$, let $\eta_{i}$ be the $i$-th marginal of $\eta$ on $\mathcal{T}_{i}$ that governs the private information for player $i$.

A behavioral strategy for player $i$ is a function $s_{i}: T_{i} \times \mathcal{A}_{i} \rightarrow[0,1]$ such that $(1) s_{i}\left(t_{i}, \cdot\right)$ is a probability measure on $\mathcal{A}_{i}$, for every $t_{i} \in T_{i}$, and $(2) s_{i}(\cdot, B)$ is $\mathcal{T}_{i}$-measurable on $T_{i}$, for every Borel set $B \in \mathcal{A}_{i}$. Given a profile of strategies $\left(s_{1}, \ldots, s_{N}\right)$, the expected payoff to player $i$ is

$$
E_{i}\left(s_{1}, \ldots, s_{I}\right)=\int_{T}\left[\int_{A_{1}} \ldots \int_{A_{N}} U_{i}(t, a) s_{1}\left(t_{1} ; d a_{1}\right) \ldots s_{N}\left(t_{N} ; d a_{N}\right)\right] \eta(d t) .
$$

In this setting, a (Bayesian-Nash) equilibrium in behavioral strategies is known to exist under the following conditions.

Theorem (Balder (1988), page 273). Suppose for each player $i=1, \cdots, N$,

1. $U_{i}$ is $\mathcal{T} \otimes \mathcal{B}(A)$-measurable on $T \times A$,

2. $U_{i}(t, \cdot)$ is continuous on $A$ for every $t \in T$,

3. $\left|U_{i}\right| \leq \varphi$ on $T \times A$ for some $\varphi \in \mathcal{L}_{1}(T, \mathcal{T}, \eta)$,

4. $A_{i}$ is a compact metric space, and 
5. $\eta$ is absolutely continuous with respect to $\eta_{1} \times \eta_{2} \times \cdots \times \eta_{N}$ (the product measure derived from the marginals).

Then there exists an equilibrium in behavioral strategies.

We show this theorem applies to a finite player version of $K L M$, as follows. Suppose there are finitely many players, indexed $i=1, \ldots, N,(N \geq 2$,$) each considering an action$ in $A_{i}=\{0,1\}$ (endowed with the discrete topology and sigma-algebra). The payoff of each player depends on an underlying state of the world $x_{0} \in \mathbb{R}$ and on the proportion of other players who play action 1 , and is given by

$$
U_{i}\left(x_{0}, a_{1}, \ldots, a_{N}\right)=\left(x_{0}+f\left(\frac{1}{N-1} \sum_{j=1, j \neq i}^{N} a_{j}\right)\right) a_{i}
$$

where $f:[0,1] \rightarrow \mathbb{R}$ is a continuous function. Similar to KLM, dominance regions are assumed as follows: there are real numbers $\underline{x}<\bar{x}$ such that for $x_{0}<\underline{x}$ the strictly dominant action for every player is to play 0 and for $x_{0}>\bar{x}$ the strictly dominant action for every player is to play 1.

The information structure is given as follows. Let $\varepsilon_{0}$ be a random variable distributed uniformly on $[\underline{x}, \bar{x}]$ with density $\phi_{0}$. Given an underlying state of the world $x_{0}$, each player $i$ observes $x_{i}=x_{0}+\varepsilon_{i}$, where $\varepsilon_{i}$ are identically distributed uniformly on $[-\epsilon, \epsilon]$, with density $\phi_{i}$, and $\left(\varepsilon_{i}\right)_{i=0}^{N}$ are jointly independent. The type space of players $i=1, \ldots, N$ is $T_{i}=[\underline{x}-\epsilon, \bar{x}+\epsilon]$ with the Borel sigma-algebra, $\mathcal{T}_{i}$. To make an explicit comparsion to Balder's theorem, we introduce player 0 ("chance" or "nature") with a trivial action space (a singleton), with type space $T_{0}=[\underline{x}, \bar{x}]$ with the Borel sigma-algebra $\mathcal{T}_{0}$, and with payoffs set identically to zero. In this case, the joint density of the information structure is given by $\phi\left(x_{0}, x_{1}, \ldots, x_{N}\right)=\phi_{0}\left(x_{0}\right) \phi_{1}\left(x_{1}-x_{0}\right) \cdots \phi_{N}\left(x_{N}-x_{0}\right)$, so that the measure $\eta$ on $\mathcal{T}$ is given by $\eta(B)=\int_{B} \phi(x) \lambda^{N+1}(d x)$, where $\lambda^{N+1}$ is Lebesgue measure on $\mathbb{R}^{N+1}$ and $B \in \mathcal{T}$.

Given a profile of strategies $\left(s_{0}, s_{1}, \ldots, s_{N}\right)$, the expected payoff to player $i=1, \ldots, N$ 
is

$$
E_{i}\left(s_{0}, s_{1}, \ldots, s_{I}\right)=\int_{T}\left[\int_{A_{1}} \ldots \int_{A_{N}} U_{i}\left(x_{0}, a_{1}, \ldots, a_{N}\right) s_{1}\left(x_{1} ; d a_{1}\right) \ldots s_{I}\left(x_{N} ; d a_{N}\right)\right] \eta(d x),
$$

and the expected payoff to player 0 is identically zero. Notice that $E_{i}$ does not depend on $s_{0}$.

Corollary 1. In the finite player version of KLM, there is an equilibrium in behavioral strategies.

Proof. Conditions (1)-(4) in Balder's theorem hold, because for each player $i, A_{i}$ is finite, $T_{i}$ is compact, and $U_{i}$ is continuous on $T \times A$. To see that (5) holds, for each $i=0,1, \ldots, N$, let the marginal density on $T_{i}$ derived from $\phi$ be given by $f_{i}\left(x_{i}\right)=\int \phi\left(x_{i}, x_{-i}\right) \lambda^{N}\left(d x_{-i}\right)$, where $\lambda^{N}$ is Lebesgue measure on $\mathbb{R}^{N}$, and the corresponding marginal measure is given by $\eta_{i}(B)=\int_{B} f_{i}\left(x_{i}\right) \lambda(d x)$, where $\lambda$ is Lebesgue measure on $\mathbb{R}$ and $B \in \mathcal{T}_{i}$. Let the product of the marginal measures be denoted $\mu=\eta_{0} \times \eta_{1} \times \cdots \times \eta_{N}$ and the product of the marginal densities be denoted $f$. Thus, $\mu(B)=\int_{B} f(x) \lambda^{N+1}(d x)$.

To see that $\eta$ is absolutely continuous with respect to $\mu$, notice that $\operatorname{support}(\phi) \subset$ $T=[\underline{x}, \bar{x}] \times[\underline{x}-\epsilon, \bar{x}+\epsilon]^{N}$. Moreover, for every $i=0, \ldots, N$ and for every $x_{i} \in T_{i}$, $f_{i}\left(x_{i}\right)>0$, and therefore, $T \subset \operatorname{support}(f)$. Fix $B \in \mathcal{T}$ arbitrarily and suppose $\mu(B)=$ $\int_{B} f(x) \lambda^{N+1}(d x)=0$. Then either $\lambda^{N+1}(B)=0$ or for $\lambda^{N+1}$-a.e. $x \in B, f(x)=0$. In the first case, $\lambda^{N+1}(B)=0$ implies that $\eta(B)=\int_{B} \phi(x) \lambda^{N+1}(d x)=0$. In the second case, for $\lambda^{N+1}$-a.e. $x \in B, f(x)=0$ implies that for $\lambda^{N+1}$-a.e. $x \in B, \phi(x)=0$, which implies that $\eta(B)=\int_{B} \phi(x) \lambda^{N+1}(d x)=0$. Consequently, $\eta$ is absolutely continuous with respect to $\mu$. An application of Balder's theorem now yields an equilibrium in behavioral strategies.

As usual, for every player $i=1, \ldots, N$, the equilibrium strategy can be extended from $T_{i}$ to $\mathbb{R}$ by playing the strictly dominant action on the complement of $T_{i}$. Notice that the equilibrium shown here is in the standard setting with an ex-ante evaluation of payoffs. The global games equilibrium is defined with an interim evaluation of payoffs. An interim equilibrium can be derived from the ex-ante equilibrium as follows. 
Consider an ex-ante equilibrium in behavioral strategies $\left(s_{0}^{*}, s_{1}^{*}, \ldots, s_{N}^{*}\right)$. For each player $i=1, \ldots, N$, define the conditional density on the types of others by $\hat{\phi}_{i}\left(x_{-i} \mid x_{i}\right)=$ $\frac{\phi\left(x_{i}, x_{-i}\right)}{f_{i}\left(x_{i}\right)}$, for $x_{i} \in T_{i}$, and the corresponding conditional measure on $\mathcal{T}_{-i}$ by $\hat{\eta}_{i}\left(B \mid x_{i}\right)=$ $\int_{B} \hat{\phi}_{i}\left(x_{-i} \mid x_{i}\right) \lambda^{N}\left(d x_{-i}\right)$. Ignoring the strategy of the chance player, we may re-write the (ex-ante) expected payoff to player $i=1, \ldots, N$ as

$$
E_{i}\left(s_{1}^{*}, \ldots, s_{N}^{*}\right)=\int_{T_{i}} E_{i}\left[s_{i}^{*}\left(x_{i}, 1\right), s_{-i}^{*} \mid x_{i}\right] \eta_{i}\left(d x_{i}\right),
$$

where $E_{i}\left[s_{i}^{*}\left(x_{i}, 1\right), s_{-i}^{*} \mid x_{i}\right]=\int_{T_{-i}} \int_{A_{-i}} U_{i}\left(x_{0}, 1, a_{-i}\right) s_{i}^{*}\left(x_{i}, 1\right) s_{-i}^{*}\left(x_{-i}, d a_{-i}\right) \hat{\eta}_{i}\left(d x_{-i} \mid x_{i}\right)$ is the (conditional) expected payoff to player $i$ from playing $s_{i}^{*}\left(x_{i}, 1\right)$ conditional on receiving signal $x_{i}$, given others are playing $s_{-i}^{*}$. Therefore, for every player $i=1, \ldots, N$ and for $\eta_{i}$-a.e. $x_{i}, E_{i}\left[\xi, s_{-i}^{*} \mid x_{i}\right]$ is maximized over $[0,1]$ at $s_{i}^{*}\left(x_{i}, 1\right)$. On the set of $\eta_{i}$-measure zero where $s_{i}^{*}\left(x_{i}, 1\right)$ is not optimal, let player $i$ re-optimize to find the payoff maximizing probability and denote it $\hat{s}_{i}\left(x_{i}, 1\right)$, and then extend $\hat{s}_{i}(\cdot, 1)$ to all of $T_{i}$ by setting it equal to $s_{i}^{*}(\cdot, 1)$ on the complement of this set.

In order to check that $\hat{s}_{i}(\cdot, 1)$ is $\mathcal{T}_{i}$-measurable, consider the set $B=\left\{x_{i} \mid \hat{s}_{i}\left(x_{i}, 1\right) \in C\right\}$, for arbitrary Borel-measurable $C \subset[0,1]$. Notice that the set $Z_{i}=\left\{x_{i} \mid s_{i}^{*}\left(x_{i}, 1\right) \neq \hat{s}_{i}\left(x_{i}, 1\right)\right\}$ is a subset of a set of $\eta_{i}$-measure zero, hence is $\mathcal{T}_{i}$-measurable and has $\eta_{i}$-measure zero. ${ }^{4}$ In particular, the set $\left\{x_{i} \mid s_{i}^{*}\left(x_{i}, 1\right) \in C, \hat{s}_{i}\left(x_{i}\right) \notin C\right\}$ is $\mathcal{T}_{i}$-measurable. Moreover, we know that the set $\left\{x_{i} \mid s_{i}^{*}\left(x_{i}, 1\right) \in C\right\}$ is $\mathcal{T}_{i}$-measurable, and therefore, the set $\left\{x_{i} \mid s_{i}^{*}\left(x_{i}, 1\right) \in C, \hat{s}_{i}\left(x_{i}, 1\right) \in C\right\}=\left\{x_{i} \mid s_{i}^{*}\left(x_{i}, 1\right) \in C\right\} \cap\left(T_{i} \backslash\left\{x_{i} \mid s_{i}^{*}\left(x_{i}, 1\right) \in C, \hat{s}_{i}\left(x_{i}, 1\right) \notin C\right\}\right)$ is $\mathcal{T}_{i}$-measurable, and consequently, the set

$$
B=\left\{x_{i} \mid s_{i}^{*}\left(x_{i}, 1\right) \in C, \hat{s}_{i}\left(x_{i}, 1\right) \in C\right\} \cup\left\{x_{i} \mid s_{i}^{*}\left(x_{i}, 1\right) \notin C, \hat{s}_{i}\left(x_{i}, 1\right) \in C\right\}
$$

is $\mathcal{T}_{i}$-measurable. Setting $\hat{s}_{i}\left(x_{i}, 0\right)=1-\hat{s}_{i}\left(x_{i}, 1\right)$, it follows that $\hat{s}_{i}$ is a behavioral strategy.

Finally, the set $Z=T_{0} \times Z_{1} \times \cdots \times Z_{N}$ is a set of $\mu$-measure zero, and therefore, of $\eta$-measure zero, whence for every player $i=1, \ldots, N, Z_{-i}$ is a set of $\hat{\eta}_{i}$-measure

\footnotetext{
${ }^{4}$ We are implicitly assuming that $\mathcal{T}_{i}$ is a complete sigma-algebra and $\eta_{i}$ is a complete measure. If not, we may replace $\mathcal{T}_{i}$ and $\eta_{i}$ by their completion, in the standard manner.
} 
zero. Therefore, for every $i=1, \ldots, N$, for every $x_{i} \in T_{i}$, and for every $\xi \in[0,1]$, $E_{i}\left[\xi, s_{-i}^{*} \mid x_{i}\right]=E_{i}\left[\xi, \hat{s}_{-i} \mid x_{i}\right]$. As $\hat{s}_{i}\left(x_{i}, 1\right)$ is optimal against $s_{-i}^{*}$, it remains optimal against $\hat{s}_{-i}$, whence $\left(\hat{s}_{1}, \ldots, \hat{s}_{N}\right)$ is an interim equilibrium in behavioral strategies.

Notice that the equilibrium shown here is for the case of (arbitrarily large but) finitely many players and the function $f$ measuring congestion effects is only assumed to be continuous (not analytic). The equilibrium may or may not be symmetric. Searching for symmetric equilibrium and/or moving to the continuum player case would require modifications to Balder's theorem. That requires additional results including delicate measurability arguments, and remains an open question. 


\section{References}

BALDER, E. J. (1988): "Generalized Equilibrium Existence Results For Games With Incomplete Information," Mathematics of Operations Research, 13, 265-276.

Carlsson, H., and E. Van Damme (1993): "Global games and equilibrium selection," Econometrica, 61(5), 989-1018.

GlicksBerG, I. L. (1952): "A further generalization of the Kakutani fixed point theorem, with application to Nash equilibrium points," Proceedings of the American Mathematical Society, 3(1), 170-174.

Karp, L., I. LeE, And R. MAson (2007): "A global game with strategic substitutes and complements," Games and Economic Behavior, 60, 155-175.

Milgrom, P. R., And R. J. Weber (1985): "Distributional Strategies For Games With Incomplete Information," Mathematics of Operations Research, 10(4), 619-632.

Monaco, A. J., And T. SABarwal (2015): "Games with strategic complements and substitutes," Economic Theory, forthcoming.

Morris, S., And H. Shin (2003): "Global games: Theory and applications," in Advances in Economics and Econometrics. Proceedings of the Eighth World Congress of the Econometric Society, pp. 56-114. Cambridge University Press.

Roy, S., And T. SABARWAL (2012): "Characterizing stability properties in games with strategic substitutes," Games and Economic Behavior, 75(1), 337-353. 